\title{
A comparison of the postoperative analgesic efficacy between epidural and intravenous analgesia in major spine surgery: a meta-analysis
}

This article was published in the following Dove Press journal: Journal of Pain Research

14 February 2017

Number of times this article has been viewed

\author{
Yichen Meng* \\ Heng Jiang* \\ Chenglin Zhang* \\ Jianquan Zhao \\ Ce Wang \\ Rui Gao \\ Xuhui Zhou
}

Department of Orthopedics, Changzheng Hospital, Second

Affiliated Hospital of Second Military

Medical University, Shanghai, People's

Republic of China

*These authors contributed equally to this work
Correspondence: Xuhui Zhou; Rui Gao Department of Orthopedics, Changzheng Hospital, Second Affiliated Hospital of Second Military Medical University, No 4 I5 Fengyang Road, Shanghai 200003, People's Republic of China Tel +86 2l 81886999

Fax: +862163520020

Email xhzhouspine@I63.com; rgaospine@163.com

\begin{abstract}
Postoperative analgesia remains a challenge for orthopedic surgeons. The aim of this meta-analysis is to compare the efficacy of epidural analgesia (EA) and intravenous patientcontrolled analgesia (IV-PCA) following major spine surgery. We searched electronic databases, including the PubMed, EMBASE, Ovid and Cochrane databases, for randomized controlled trials (RCTs) published before June 2016. The quality of the included trials was assessed using the Cochrane risk-of-bias tool. Random effects models were used to estimate the standardized mean differences (SMDs) and relative risks (RRs), with the corresponding 95\% confidence intervals (CI). Subgroup analyses stratified by the type of epidural-infused medication and epidural delivery were also performed. A total of 17 trials matched the inclusion criteria and were chosen for the following meta-analysis. Overall, EA provided significantly superior analgesia, higher patient satisfaction and decreased overall opioid consumption compared with IV-PCA following major spine surgery. Additionally, no differences were found in the side effects associated with these two methods of analgesia. Egger's and Begg's tests showed no significant publication bias. We suggest that EA is superior to IV-PCA for pain management after major spine surgery. More large-scale, high-quality trials are needed to verify these findings.
\end{abstract}

Keywords: adolescent idiopathic scoliosis, lumbar fusion, epidural analgesia, intravenous application, perioperative pain

\section{Introduction}

Postoperative pain is not only a matter of worsened patient-oriented outcomes, such as quality of life, it also substantially affects recovery, increases postoperative morbidities and prolongs the length of hospital stay. ${ }^{1-3}$ For patients undergoing major spine surgeries, postoperative pain typically lasts for at least 3 days. ${ }^{4}$ Although multiple analgesic strategies are used, no consensus has been established. Additionally, postoperative analgesia remains a challenging problem for orthopedic surgeons. In recent years, epidural analgesia (EA) and intravenous patient-controlled analgesia (IV-PCA) have both been identified as effective methods of postoperative pain control following spinal surgery, and the efficacies of pain control of these two methods have been compared by several randomized controlled trials (RCTs). However, despite some individual studies demonstrating the analgesic benefits of EA, no published systematic review has examined which method is better. We searched all RCTs comparing the analgesic effects of EA and IV-PCA following spinal surgeries and conducted this meta-analysis to compare EA and IV-PCA and to clarify which method is better in terms of pain control and adverse effects. 


\section{Methods}

\section{Search strategy}

This systematic review and meta-analysis was based on the Preferred Reporting Items for Systematic Reviews and MetaAnalyses (PRISMA) guidelines. ${ }^{5}$ We searched MEDLINE, EMBASE, Cochrane Library and Ovid to retrieve related studies published before June 2016 with combinations of the following keywords: "epidural", "intravenous", "spine surgery (lumbar fusion/lumbar laminectomy/scoliosis correction/spinal fracture fixation)", "postoperative", "pain", "efficacy", "analgesia" and "randomized controlled trial". The language was restricted to English and Chinese. A manual search was also conducted to identify relevant publications from the citation lists of the included articles.

\section{Inclusion and exclusion criteria}

Trials were included if they met the following criteria: 1) the study was an RCT; 2) patients received major spine surgeries, including lumbar fusion, lumbar laminectomy, scoliosis correction and spinal fracture fixation; 3) EA was defined as a medication delivered into the epidural space by infusion, a patient-controlled device or repeated bolus dosing; 6 4) the relative risk (RR) with $95 \%$ confidence interval $(95 \% \mathrm{CI}$; or crude data that allowed their calculation) were reported; and 5) the language was restricted to English and Chinese.

The following types of studies were excluded: 1) case series, case reports, reviews and observational studies; 2) studies incorporating the use of nonpharmacologic interventions as a part of the analgesic regimen; 3) studies for which the crude data were not given or the odds ratio (OR) or RR could not be calculated; and 4) publications that were not written in English or Chinese.

\section{Data extraction and study quality assessment}

Two authors independently read the full texts and extracted relevant data from each publication. The following data were extracted: first author, year of publication, country, research design, number of participants, male/female ratio, analgesic regimen used and the postoperative pain scores. Data regarding analgesia-related complications, including nausea, vomiting, pruritus and paresthesia, were also extracted. Graphical data were converted to numerical data independently by the two authors, and disagreements were resolved by consensus.

The quality of the included trials was assessed by two independent reviewers using the Cochrane risk-of-bias tool for RCTs. ${ }^{7}$ The bias domains that were evaluated with this tool included selection, performance, detection, attrition and reporting bias.

\section{Statistical analysis}

Statistical analyses were performed using Stata version 12.0 (Stata Corporation, College Station, TX, USA). We analyzed the RRs with $95 \%$ CIs for dichotomous variables and the standardized mean difference (SMD) with $95 \%$ CIs for continuous variables. Adjusted risk estimates were also abstracted. The unadjusted risk estimates were calculated using original data when the adjusted risk estimates were unavailable. The $I$-squared $\left(I^{2}\right)$ statistic was used to assess statistical heterogeneity among the studies. A value of $P^{2}>50 \%$ reflected significant heterogeneity. ${ }^{8}$ Both fixed and random effects models were used to pool the data in our study. However, the random effects model is preferable for surgical research because patients who are operated on in different centers have varying risk profiles and selection criteria for each surgical technique. ${ }^{9}$ Considering the possible strong heterogeneity resulting from potential differences across the trials, we performed a subgroup analysis stratified by medication type (opioids alone, local anesthetic alone and local anesthetic with an opioid) and epidural delivery type (patient-controlled analgesia and continuous infusion analgesia) for outcomes that were reported by at least two trials. The robustness of the combined results was evaluated by a sensitivity analysis; namely, the analysis was repeated after the exclusion of one study at a time. ${ }^{10}$ Begg's funnel plot and Egger's test for possible publication bias were used in analyses that included $>10$ studies.

\section{Results}

A total of 206 articles were identified after the initial search. After the exclusion of 128 articles that were irrelevant to our research, 78 articles remained. In total, 28 articles were retrieved for full-text review, and 20 studies were identified. Finally, three studies were excluded because the crude data could not be extracted. Therefore, 17 trials matched the inclusion criteria and were chosen for the following metaanalysis (Figure 1). These trials were dated from 1989 to 2016, had prospective randomized designs and included a total of 938 participants, of which 508 (54.2\%) received EA and 430 (45.8\%) underwent IV-PCA. The characteristics of the retrieved studies are presented in Table 1. Of the 17 included trials, eight trials enrolled patients undergoing lumbar fusion, ${ }^{11-18}$ six enrolled patients undergoing adolescent idiopathic scoliosis (AIS) correction ${ }^{19-24}$ and three enrolled patients undergoing other major spine surgeries. ${ }^{25-27}$ 


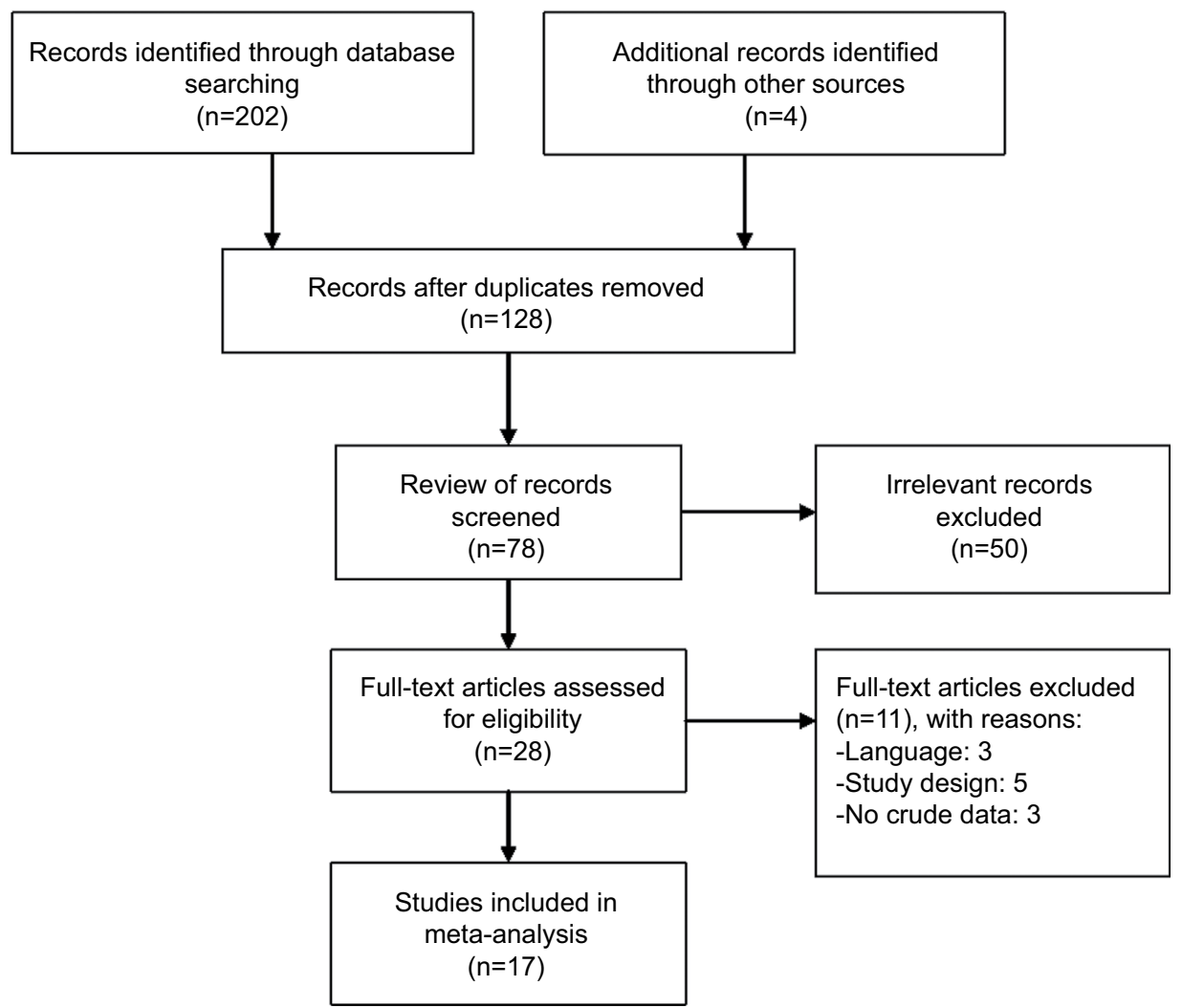

Figure I Flow chart of the process of screening of studies.

All IV-PCA patients received opioids for pain control. For EA regimens, $12.2 \%$ of the epidural patients received opioids alone, whereas $14.8 \%$ received a local anesthetic alone and $73 \%$ received a local anesthetic and opioid. A study by Prasartritha et $\mathrm{a}^{27}$ compared three regimens (epidural opioids, epidural opioids and a local anesthetic, and intravenous opioids), and we divided this trial into two comparisons (epidural opioids versus intravenous opioids; and epidural opioids and a local anesthetic versus intravenous opioids) in the following analysis. For the application of epidural analgesics, six studies utilized patient-controlled epidural analgesia (PCEA), and 11 studies utilized continuous epidural infusion analgesia (CEIA). Blumenthal et al ${ }^{19,20}$ compared the efficacy of two types of epidural catheter infusion with intravenous infusion, while the other studies compared the efficacy of a single type of epidural catheter infusion with intravenous infusion. Klatt et $\mathrm{al}^{23}$ compared three delivery techniques (a single catheter, double catheter and intravenous), and we divided this trial into two comparisons (single catheter versus intravenous delivery, and double catheter versus intravenous delivery). More than half of the included trials described the randomization methods (a computerized list or random number table). All studies reported detailed information about withdrawal and dropouts. Blinding is not a necessity in our analysis because the placement of a sham epidural catheter that is not used for pain management is unequivocally not considered to be ethical. ${ }^{28}$ Therefore, the methodology of all included studies could be considered to be of moderate-to-high quality. The results of the quality assessment with the Cochrane risk-of-bias checklist are summarized in Figures S1 and S2.

\section{Pain}

Postoperative pain scores were recorded using a $0-10 \mathrm{~cm}$ visual analog scale (VAS), on which a score of zero indicates no pain and a score of 10 indicates the worst conceivable pain. ${ }^{29}$ Fourteen studies reported postoperative VAS scores. ${ }^{11,12,14-22,24-26}$ The meta-analysis results demonstrated that, overall, EA provided significantly superior analgesic effects compared with IV-PCA at all time points.

On postoperative day 1, epidural patients in the lumbar surgery group had a lower VAS score than IV-PCA patients ( $p=0.029, \mathrm{SMD}=-1.15,95 \% \mathrm{CI}=-2.19$ to -0.12$)$. For patients undergoing scoliosis correction or other spine surgeries, no significant difference was noted between EA and IV-PCA (Figure 2). 


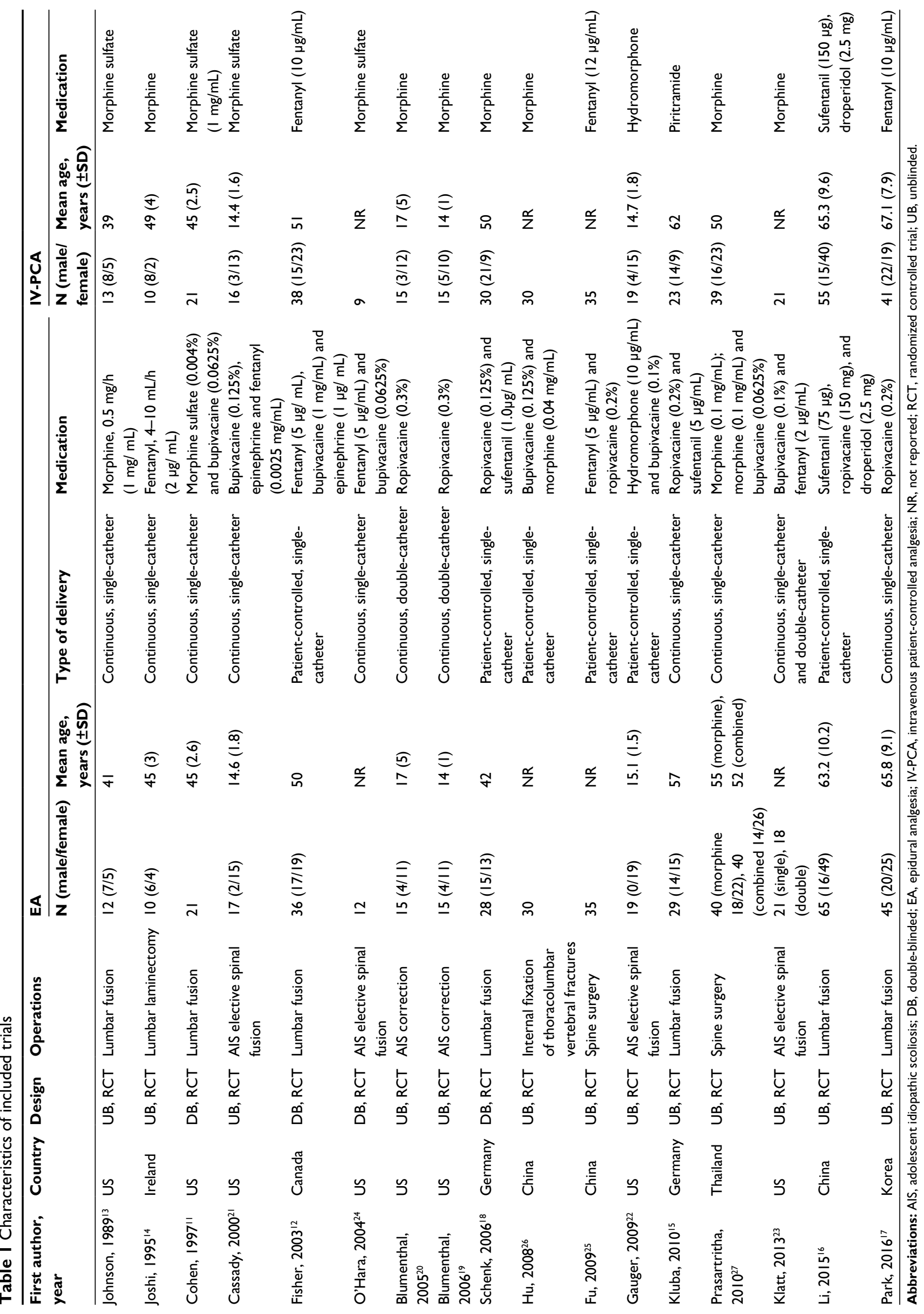


Study

ID

\section{Lumbar surgery}

Joshi et al ${ }^{14}$

Cohen et al $^{11}$

Fisher et al ${ }^{12}$

Schenk et al ${ }^{18}$

Kluba et $\mathrm{al}^{15}$

Li et $\mathrm{al}^{16}$

Park et al ${ }^{17}$

Subtotal $(I$-squared $=95.7 \%, p=0.000)$

Scoliosis correction

Cassady et $\mathrm{al}^{21}$

O'Hara et al ${ }^{24}$

Blumenthal et $\mathrm{al}^{20}$

Blumenthal et $\mathrm{al}^{19}$

Gauger et $\mathrm{al}^{22}$

Subtotal $(I$-squared $=74.4 \%, p=0.004)$

Others

Hu et al ${ }^{26}$

Fu et $\mathrm{al}^{25}$

Subtotal $(I$-squared $=96.6 \%, p=0.000$ )

Overall $(I$-squared $=93.0 \%, p=0.000)$

Note: Weights are from random effects analysis

$$
\begin{gathered}
1 \\
-5.57
\end{gathered}
$$

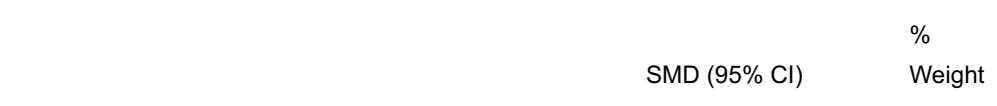




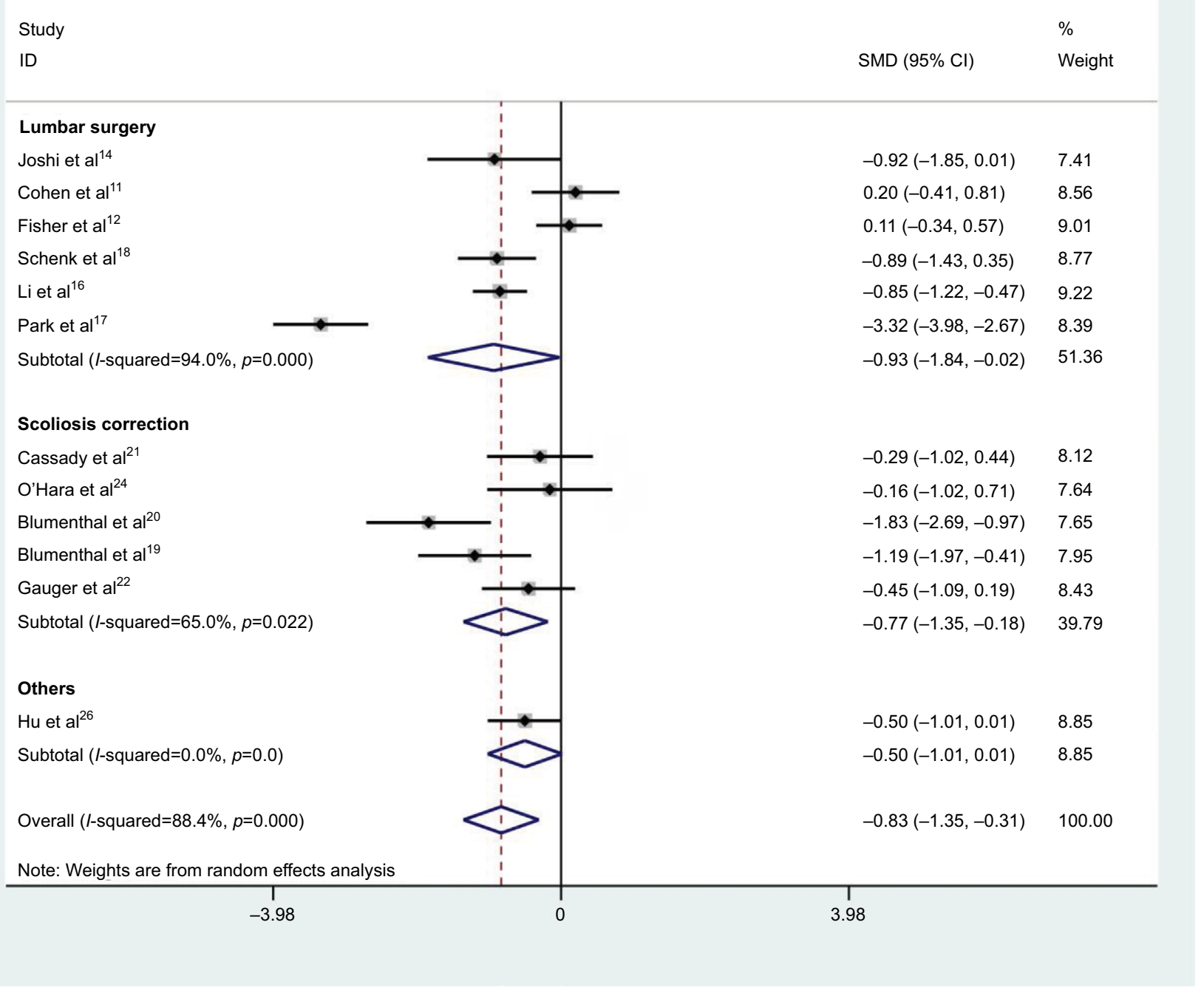

Figure 3 Summary effect of postoperative pain scores 48 hours after surgery.

Notes: Diamonds represent pooled estimates, and width of the diamonds represents $95 \% \mathrm{Cls}$. The $p$-value was calculated using chi-squared test.

Abbreviations: $\mathrm{Cl}$, confidence interval; SMD, standardized mean difference.

\section{Pruritus}

Nine trials, including 11 comparisons, reported the incidence of pruritus, ${ }^{11,12,16-18,21,23,26,27}$ with two showing a statistically significant reduction in the EA group compared with the IV-PCA group. ${ }^{17,26}$ The meta-analysis of all trials did not reveal any significant difference between the groups $(p=0.23$, $\mathrm{RR}=1.52,95 \% \mathrm{CI}=0.77$ to 2.99 for subcategory lumbar fusion; $p=0.27, \mathrm{RR}=1.26,95 \% \mathrm{CI}=0.84$ to 1.91 for the scoliosis correction subcategory) (Figure S6). When the lumbar fusion trials were stratified by the epidural delivery type, no significant difference in pruritus was observed (Figure S7). Furthermore, in the subgroup stratified by the type of epidural medication (Figure S8), epidural-infused local anesthetic with an opioid had a borderline elevated risk for the incidence of pruritus ( $p=0.051, \mathrm{RR}=1.67,95 \%$ $\mathrm{CI}=1.00$ to 2.78 ).

\section{Paresthesia}

Four studies reported the incidence of paresthesia following postoperative analgesia, ${ }^{12,15,22,23}$ with none individually showing a statistically significant reduction in the EA versus IV-PCA groups. The meta-analysis of all studies also demonstrated no difference between these two analgesic methods for different surgeries $(p=0.054, \mathrm{RR}=7.56,95 \%$ $\mathrm{CI}=0.97$ to 59.17 for lumbar fusion; $p=0.45$, $\mathrm{RR}=1.60$, $95 \% \mathrm{CI}=0.48$ to 5.34 for scoliosis correction) (Figure S9).

\section{The amount of opioids used}

The amount of perioperative opioids used was reported in seven studies. ${ }^{11,12,14,17,19,20,24}$ O'Hara et al ${ }^{24}$ reported no difference in morphine consumption between the groups but provided no detailed data, and therefore this study was excluded from the analysis. Pooled data from the other six studies 
Study

ID $\begin{array}{ll}\% \\ \operatorname{SMD}(95 \% \mathrm{Cl}) & \text { Weight }\end{array}$

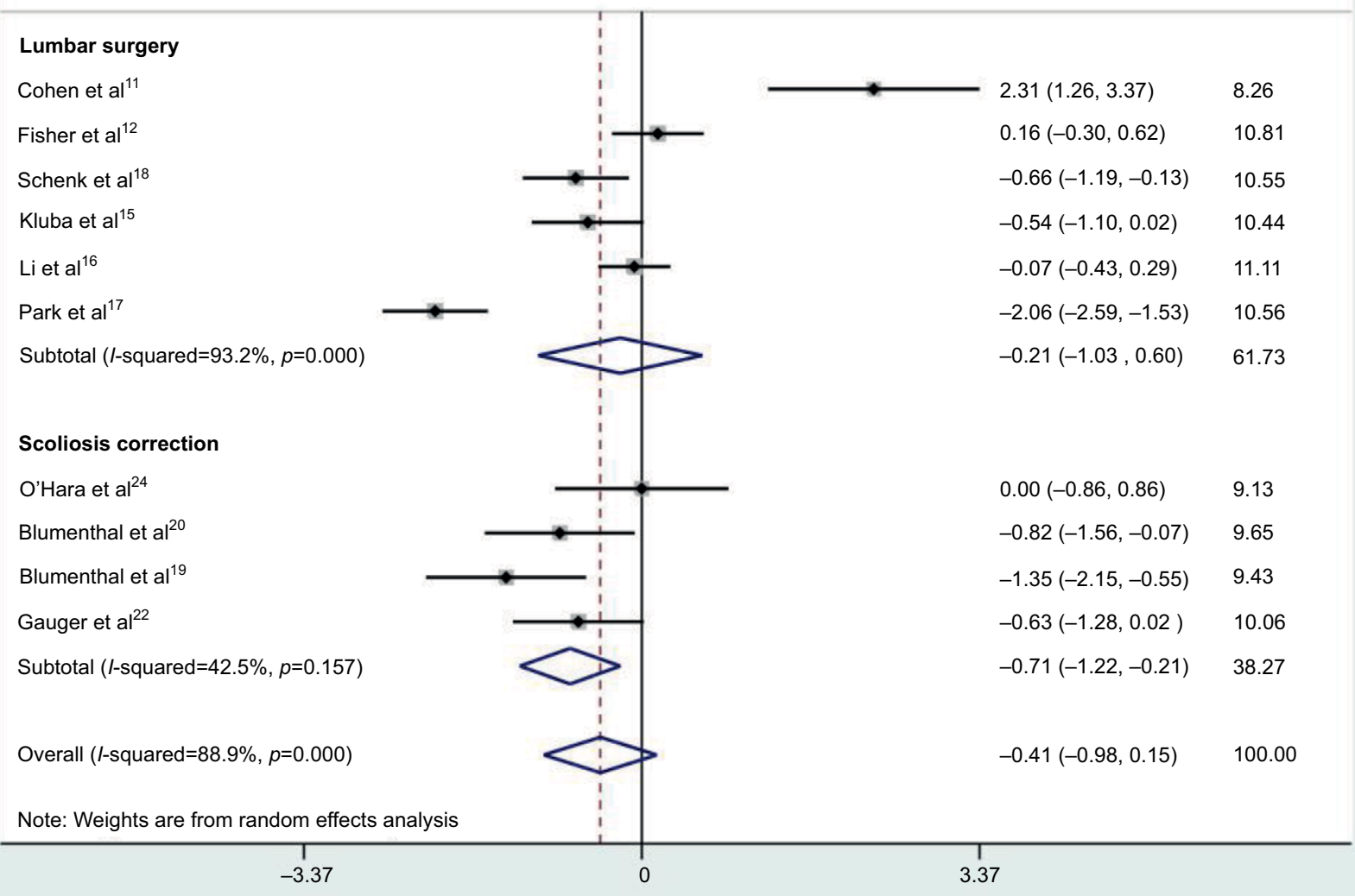

Figure 4 Summary effect of postoperative pain scores 72 hours after surgery.

Notes: Diamonds represent pooled estimates, and width of the diamonds represents $95 \% \mathrm{Cls}$. The $p$-value was calculated using chi-squared test.

Abbreviations: $\mathrm{Cl}$, confidence interval; SMD, standardized mean difference.

revealed a significantly reduced amount of opioids in the EA group. The results were as follows: $p=0.012, \mathrm{SMD}=-3.12$, $95 \% \mathrm{CI}=-5.56$ to -0.68 for the lumbar fusion subcategory; and $p<0.01$, SMD $=-1.45,95 \% \mathrm{CI}=-2.02$ to -0.87 for the scoliosis correction subcategory (Figure S10).

\section{Patient satisfaction}

Five of 17 studies assessed patient satisfaction with postoperative pain management, and all of them applied a numeric scale, with a higher score indicating higher satisfaction. ${ }^{12,15,17,19,20}$ The results showed that patients undergoing scoliosis correction were significantly more satisfied in the EA group ( $p<0.01, \mathrm{SMD}=2.25,95 \% \mathrm{CI}=1.59$ to 2.90 ), whereas the satisfaction of patients undergoing lumbar fusion did not significantly differ between treatment types ( $p=0.09$, $\mathrm{SMD}=0.39,95 \% \mathrm{CI}=-0.06$ to 0.84 ) (Figure $\mathrm{S} 11$ ).

\section{Motor block}

Incidence of motor block was reported by two studies. ${ }^{17,18}$ Both studies compared the efficacy of EA and IV-PCA following lumbar fusion. The results indicated that patients in the EA group had a 15 times risk of experiencing motor block ( $p=0.008, \mathrm{RR}=15.07,95 \% \mathrm{CI}=2.04$ to 111.34 ) (Figure S12).

\section{Publication bias and sensitivity analysis}

A funnel plot of the logarithm of RR $(\log R R)$ against the logarithm of standard error was constructed for the analysis of the incidence of nausea and vomiting, in which 13 comparisons were included (Figure 5). Visual inspection of the funnel plots did not show any remarkable asymmetry. In addition, Egger's and Begg's tests showed no significant publication bias ( $p=0.179$ for Egger's test and $p=0.502$ 


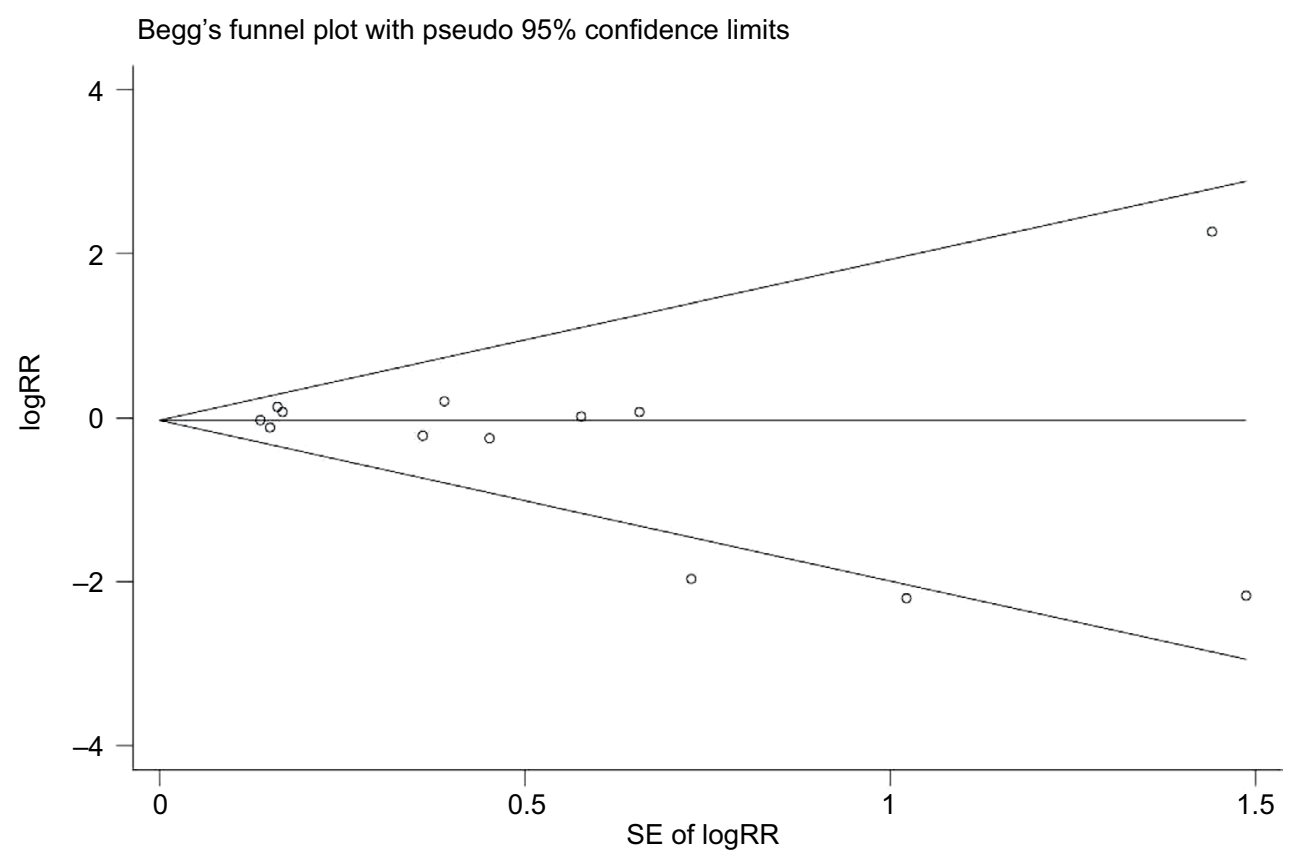

Figure 5 Funnel plot for the meta-analysis on the incidence of nausea and vomiting. Notes: Each point represents a separate study for the indicated association. The horizontal line indicates the effect size. Abbreviations: $\mathrm{SE}$, standard error; RR, risk ratio; logRR, natural logarithm of risk ratio.

for Begg's test). A sensitivity analysis was conducted by excluding each study one by one, and the pooled results were not significantly attenuated, which confirmed the robustness of the data (Figure S13).

\section{Discussion}

Severe pain is encountered by most patients undergoing major spine surgery. EA and IV-PCA are two common options for treating postoperative pain, and previous systematic reviews have shown that EA provides superior pain management. ${ }^{6,30}$ However, both systematic reviews enrolled small numbers of patients undergoing spine surgery, and no universally agreed-upon consensus exists regarding which analgesic approach performs better in spine surgery patients. ${ }^{22}$ In the literature, Joshi et $\mathrm{al}^{14}$ compared the efficacy and safety of EA with IV-PCA in the management of postoperative pain after lumbar laminectomy and found that EA was superior for controlling pain. However, in a prospective randomized double-blind clinical trial conducted by Cohen et al, ${ }^{11}$ the data suggested no clinical advantage of EA over IV-PCA for spinal fusion patients. A trial by Cassady et $\mathrm{al}^{21}$ demonstrated that EA and IV-PCA were comparably effective and safe for scoliosis correction procedures in adolescents, which was consistent with the findings reported by O'Hara et al, ${ }^{24}$ but was in contrast to the findings reported by Gauger et al, ${ }^{22}$ who found EA to be more effective than IV-PCA for pain relief. These discrepancies are likely due to the relatively small sample sizes, which provided limited statistical power.
Therefore, we performed this meta-analysis to comprehensively evaluate the ability of EA and IV-PCA to improve pain control following major spine surgeries.

We conducted an analysis stratified by surgery type, and for patients undergoing scoliosis correction, no difference was found for the use of EA and IV-PCA for pain control on postoperative day 1 . However, on postoperative days 2 and 3 , EA significantly alleviated pain compared to IV-PCA. In contrast, for patients undergoing lumbar fusion, EA provided better pain management than IV-PCA on postoperative days 1 and 2 but failed to have a superior analgesic effect on day 3. We believe this difference was due to two reasons. First, patients undergoing scoliosis correction were all children and adolescents, while patients undergoing lumbar fusion were mostly adults with degenerative spinal diseases. Additionally, adolescents have been reported to have more pain than adults after surgery. ${ }^{31}$ Second, the researchers adjusted their analgesic doses daily to facilitate patient comfort, which may influence the results. ${ }^{22}$ When we further investigated the effects of the type of epidural delivery on our results, we found that CEIA provided better pain control following scoliosis correction at all time points. For patients undergoing lumbar fusion, neither CEIA nor PCEA had better analgesic effects than IV-PCA. This result was not consistent with the research presented by Lu et al, ${ }^{32}$ which confirmed that PCEA was more effective in relieving pain than IV-PCA for spinal fusion patients. The explanation for this discrepancy may be as follows. First, Lu et $\mathrm{al}^{32}$ grouped patients undergoing 
scoliosis correction and patients undergoing lumbar fusion together, which led to exaggerated results. Second, they calculated the OR for dichotomous variables and used a fixedeffect model in their analysis. In contrast, we considered it more appropriate to calculate the RR and to use a random effects model with RCTs, which is the more conservative approach. ${ }^{33}$ Furthermore, we evaluated the effects of epidural medications on our results. Surprisingly, EA with opioids and local anesthetics did not provide a significant benefit for different types of surgery. However, local anesthetic-based EA significantly relieved pain following scoliosis correction surgery. Both related trials used ropivacaine as the epiduralinfused drug. ${ }^{19,20}$ However, another local anesthetic, bupivacaine, failed to support the better analgesic effect of epidurals compared with IV-PCA. ${ }^{21}$ This lack of an analgesic effect may have been related to the lower dosage of bupivacaine used due to safety concerns. ${ }^{34}$ Compared to bupivacaine, ropivacaine has been suggested to significantly improve safety profiles and to provide effective pain relief at lower concentrations. ${ }^{35}$ Recently, Park et $\mathrm{al}^{17}$ conducted a prospective comparative trial including 94 patients harboring lumbar spinal stenosis. The patients were randomized to receive either EA with ropivacaine (51 patients) or conventional IV-PCA (43 patients). During the first postoperative 3 days, significantly lower pain scores were observed in the EA group. However, the benefits of ropivacaine-based EA should be interpreted with caution due to the limited number and small sample sizes of relevant trials. The number of epidural catheters has also been postulated to influence the analgesic effects. Blumenthal et al ${ }^{19,20}$ compared the analgesic effects of two intraoperatively placed epidural catheters with IV-PCA, and the results indicated that the double epidural catheter technique provided better postoperative analgesia. In another trial, Klatt et al compared single and double epidural catheters with IV-PCA. The results demonstrated that while single epidural analgesia and IV-PCA provided good pain control, double epidural catheter analgesia provided consistently better pain control than either the single epidural catheter technique or IV-PCA. ${ }^{23}$ Both trials were conducted in adolescent patients, and future studies are needed to confirm whether this double-catheter technique would also be beneficial in adult patients.

Regarding the analgesia-related side effects, we did not find any significant difference between EA and IV-PCA. The overall side effect rates reported were comparable to those observed by other sources. The cumulative incidence of nausea and vomiting from EA was reported to be $27.6 \%$, and the incidence of pruritus was $32.8 \% .^{36}$ In our analysis, the percentages of nausea and vomiting and pruritus were $28.1 \%$ and $23.7 \%$, respectively.
Motor block after spine surgery, which may confound the diagnosis of neurodeficits due to the surgery itself, was regarded as one of most important issues in several trials. ${ }^{17,18}$ Motor block was not reported after administration of $10 \mathrm{~mL}$ of ropivacaine $(0.1 \%)$ by Gottschalk et al. ${ }^{37}$ In a trial by Schenk et al, ${ }^{18}$ motor block occurred after the initial bolus of $14 \mathrm{~mL}$ of $0.125 \%$ ropivacaine in five of 28 patients $(17.9 \%)$ in the EA group. In a study by Park et al, ${ }^{17}$ an epidural infusion of ropivacaine $(0.2 \%)$ caused a transient motor block in 10 of 47 patients $(22 \%)$; however, reducing the infusion rate led to the return of motor function. Thus, the optimal concentration and infusion rate of local anesthetics should be considered before beginning epidural pain management.

This analysis has some limitations. First, publication bias cannot be excluded because only publications in English and Chinese were included in our analysis. ${ }^{38}$ A possibility exists that we missed certain eligible studies. Second, separate evaluations of pain at rest and with activity have become the more accepted method for evaluating pain. ${ }^{22}$ Most of the included trials failed to use this method, which may attenuate the pain levels. Third, one must consider the validity and difficulty in measuring outcomes such as pain levels among adolescent patients, which was the main reason that we divided the trials according to the type of surgery and chose the SMD as the estimate. Fourth, the sample sizes of the included studies were relatively small, which led to a possible type II error and therefore limited the power of our results. Finally, 13 of 17 included trials used an unblended design in the allocation of treatment and the assessment of outcomes.

\section{Conclusion}

Despite these limitations, the results of this meta-analysis suggest that EA has several advantages over IV-PCA with respect to pain management, patient satisfaction and overall opioid consumption after major spine surgery. No difference was observed in the side effects associated with these two analgesic methods. Continuous EA is superior for controlling pain in adolescents undergoing scoliosis correction. Local anesthetics seem to be candidate drugs for epidural regimens in the future. More large-scale, high-quality trials that consider multiple variables (ie, types of medication infused, the optimal infusion rate, optimal medication concentration and the number of catheters) are warranted to verify these findings.

\section{Acknowledgments}

We would like to thank the authors of the original studies included in this meta-analysis. No funding was received for this work. 


\section{Disclosure}

The authors report no conflicts of interest in this work.

\section{References}

1. Kehlet H. Postoperative pain relief - what is the issue? Br J Anaesth. 1994;72(4):375-378.

2. Yukawa Y, Kato F, Ito K, Terashima T, Horie Y. A prospective randomized study of preemptive analgesia for postoperative pain in the patients undergoing posterior lumbar interbody fusion: continuous subcutaneous morphine, continuous epidural morphine, and diclofenac sodium. Spine (Phila Pa 1976). 2005;30(21):2357-2361.

3. Carli F, Mayo N, Klubien K, Schricker T, Trudel J, Belliveau P. Epidural analgesia enhances functional exercise capacity and health-related quality of life after colonic surgery: results of a randomized trial. Anesthesiology. 2002;97(3):540-549.

4. Bianconi M, Ferraro L, Ricci R, et al. The pharmacokinetics and efficacy of ropivacaine continuous wound instillation after spine fusion surgery. Anesth Analg. 2004;98(1):166-172. table of contents.

5. Moher D, Liberati A, Tetzlaff J, Altman DG. Preferred reporting items for systematic reviews and meta-analyses: the PRISMA statement. $B M J$. 2009;339:b2535.

6. Block BM, Liu SS, Rowlingson AJ, Cowan AR, Cowan JA Jr, Wu CL. Efficacy of postoperative epidural analgesia: a meta-analysis. JAMA. 2003;290(18):2455-2463.

7. Higgins JP, Sally G. Cochrane Handbook for Systematic Reviews of Interventions. 2011. Available from: http://handbook.cochrane.org/. Accessed April 1, 2012.

8. Higgins JP, Thompson SG, Deeks JJ, Altman DG. Measuring inconsistency in meta-analyses. BMJ. 2003;327(7414):557-560.

9. Aziz O, Athanasiou T, Tekkis PP, et al. Laparoscopic versus open appendectomy in children: a meta-analysis. Ann Surg. 2006;243(1):17-27.

10. Patsopoulos NA, Evangelou E, Ioannidis JP. Sensitivity of betweenstudy heterogeneity in meta-analysis: proposed metrics and empirical evaluation. Int J Epidemiol. 2008;37(5):1148-1157.

11. Cohen BE, Hartman MB, Wade JT, Miller JS, Gilbert R, Chapman TM. Postoperative pain control after lumbar spine fusion. Patient-controlled analgesia versus continuous epidural analgesia. Spine (Phila Pa 1976). 1997;22:1892-1896. discussion 1896-1897.

12. Fisher CG, Belanger L, Gofton EG, et al. Prospective randomized clinical trial comparing patient-controlled intravenous analgesia with patient-controlled epidural analgesia after lumbar spinal fusion. Spine (Phila Pa 1976). 2003;28(8):739-743.

13. Johnson RG, Miller M, Murphy M. Intraspinal narcotic analgesia. A comparison of two methods of postoperative pain relief. Spine (Phila Pa 1976). 1989;14(4):363-366.

14. Joshi GP, McCarroll SM, O'Rourke K. Postoperative analgesia after lumbar laminectomy: epidural fentanyl infusion versus patient-controlled intravenous morphine. Anesth Analg. 1995;80(3):511-514.

15. Kluba T, Hofmann F, Bredanger S, Blumenstock G, Niemeyer T. Efficacy of post-operative analgesia after posterior lumbar instrumented fusion for degenerative disc disease: a prospective randomized comparison of epidural catheter and intravenous administration of analgesics. Orthop Rev (Pavia). 2010;2(1):e9.

16. Li Y, Lu S, Ma SC, Fan HW, Zhao GQ. Effects of patient-controlled epidural analgesia and patient-controlled intravenous analgesia on analgesia in patients undergoing spinal fusion surgery. Am $J$ Ther. 2016;23(6):e1806-e1812.

17. Park SY, An HS, Lee SH, Suh SW, Kim JL, Yoon SJ. A prospective randomized comparative study of postoperative pain control using an epidural catheter in patients undergoing posterior lumbar interbody fusion. Eur Spine J. 2016;25(5):1601-1607.

18. Schenk MR, Putzier M, Kügler B, et al. Postoperative analgesia after major spine surgery: patient-controlled epidural analgesia versus patientcontrolled intravenous analgesia. Anesth Analg. 2006;103(5):1311-1317.
19. Blumenthal S, Borgeat A, Nadig M, Min K. Postoperative analgesia after anterior correction of thoracic scoliosis: a prospective randomized study comparing continuous double epidural catheter technique with intravenous morphine. Spine (Phila Pa 1976). 2006;31(15):1646-1651.

20. Blumenthal S, Min K, Nadig M, Borgeat A. Double epidural catheter with ropivacaine versus intravenous morphine: a comparison for postoperative analgesia after scoliosis correction surgery. Anesthesiology. 2005;102(1):175-180.

21. Cassady JF Jr, Lederhaas G, Cancel DD, Cummings RJ, Loveless EA. A randomized comparison of the effects of continuous thoracic epidural analgesia and intravenous patient-controlled analgesia after posterior spinal fusion in adolescents. Reg Anesth Pain Med. 2000;25(3): 246-253.

22. Gauger VT, Voepel-Lewis TD, Burke CN, et al. Epidural analgesia compared with intravenous analgesia after pediatric posterior spinal fusion. J Pediatr Orthop. 2009;29(6):588-593.

23. Klatt JW, Mickelson J, Hung M, Durcan S, Miller C, Smith JT. A randomized prospective evaluation of 3 techniques of postoperative pain management after posterior spinal instrumentation and fusion. Spine (Phila Pa 1976). 2013;38(19):1626-1631.

24. O’Hara JF Jr, Cywinski JB, Tetzlaff JE, Xu M, Gurd AR, Andrish JT. The effect of epidural vs intravenous analgesia for posterior spinal fusion surgery. Paediatr Anaesth. 2004;14(12):1009-1015.

25. Fu CQ, Cao DQ. Effects of patient-controlled epidural analgesia and patient-controlled intravenous analgesia on analgesia in patients undergoing spinal surgery. J Clin Res. 2009;26:123-125.

26. Hu MP, Li XW, Lian QQ, Chen LY, Mao FM. Effects of different analgesic methods on postoperative pain in internal fixation patients with thoracolumbar vertebrae fractures. Herald Med. 2008;27:661-663.

27. PrasartrithaT, Kunakornsawat S, Tungsiripat R, Jampa J, Throngnumchai R. A prospective randomized trial comparing epidural morphine through intraoperatively placed epidural catheter and intravenous morphine in major lumbar spinal surgery. J Spinal Disord Tech. 2010;23(8): e43-e46.

28. Taenzer AH, Clark C. Efficacy of postoperative epidural analgesia in adolescent scoliosis surgery: a meta-analysis. Paediatr Anaesth. 2010; 20(2):135-143.

29. Wong DL, Baker CM. Smiling faces as anchor for pain intensity scales. Pain. 2001;89(2-3):295-300.

30. Dolin SJ, Cashman JN, Bland JM. Effectiveness of acute postoperative pain management: I. Evidence from published data. Br J Anaesth. 2002;89(3):409-423.

31. Gillies ML, Smith LN, Parry-Jones WL. Postoperative pain assessment and management in adolescents. Pain. 1999;79(2-3):207-215.

32. Lu S, Ma SC, Wang YY, Zhu ZH, Fan HW, Zhao GQ. Comparison of pain relief between patient-controlled epidural analgesia and patientcontrolled intravenous analgesia for patients undergoing spinal fusion surgeries. Arch Orthop Trauma Surg. 2015;135(9):1247-1255.

33. Berlin JA, Laird NM, Sacks HS, Chalmers TC. A comparison of statistical methods for combining event rates from clinical trials. Stat Med. 1989;8(2):141-151.

34. Zink W, Seif C, Bohl JR, et al. The acute myotoxic effects of bupivacaine and ropivacaine after continuous peripheral nerve blockades. Anesth Analg. 2003;97(4):1173-1179. table of contents.

35. Whiteside JB, Wildsmith JA. Developments in local anaesthetic drugs. Br J Anaesth. 2001;87(1):27-35.

36. Wu CL, Cohen SR, Richman JM, et al. Efficacy of postoperative patient-controlled and continuous infusion epidural analgesia versus intravenous patient-controlled analgesia with opioids: a meta-analysis. Anesthesiology. 2005;103(5):1079-1088. quiz 1109-1010.

37. Gottschalk A, Freitag M, Tank S, et al. Quality of postoperative pain using an intraoperatively placed epidural catheter after major lumbar spinal surgery. Anesthesiology. 2004;101(1):175-180.

38. Gregoire G, Derderian F, Le Lorier J. Selecting the language of the publications included in a meta-analysis: is there a Tower of Babel bias? J Clin Epidemiol. 1995;48(1):159-163. 
The Journal of Pain Research is an international, peer reviewed, open access, online journal that welcomes laboratory and clinical findings in the fields of pain research and the prevention and management of pain. Original research, reviews, symposium reports, hypothesis formation and commentaries are all considered for publication
The manuscript management system is completely online and includes a very quick and fair peer-review system, which is all easy to use. Visit http://www.dovepress.com/testimonials.php to read real quotes from published authors. 